\title{
Search for a Variation of the Fine Structure Constant around the Supermassive Black Hole in Our Galactic Center
}

\author{
A. Hees $\odot,{ }^{1, *}$ T. Do $\odot,{ }^{2}$ B. M. Roberts $\odot,{ }^{1,3}$ A. M. Ghez, ${ }^{2}$ S. Nishiyama $\odot,{ }^{4}$ R. O. Bentley $\odot,{ }^{2}$ A. K. Gautam $\odot,{ }^{2}$ \\ S. Jia $\odot,{ }^{5}$ T. Kara, ${ }^{4}$ J. R. Lu $\odot,{ }^{5}$ H. Saida $\odot,{ }^{6}$ S. Sakai $\odot,{ }^{2}$ M. Takahashi $\odot,{ }^{7}$ and Y. Takamori ${ }^{8}$ \\ ${ }^{1}$ SYRTE, Observatoire de Paris, Université PSL, CNRS, Sorbonne Université, \\ LNE, 61 avenue de l'Observatoire 75014 Paris, France \\ ${ }^{2}$ Department of Physics and Astronomy, University of California, Los Angeles, California 90095, USA \\ ${ }^{3}$ School of Mathematics and Physics, The University of Queensland, Brisbane, Queensland 4072, Australia \\ ${ }^{4}$ Miyagi University of Education, 149 Aramaki-aza-aoba, Aoba-ku, Sendai, Miyagi 980-0845, Japan \\ ${ }^{5}$ Astronomy Department, University of California, Berkeley, California 94720, USA \\ ${ }^{6}$ Daido University, 10-3 Takiharu-cho, Minami-ku, Nagoya, Aichi 457-8530, Japan \\ ${ }^{7}$ Aichi University of Education, 1 Hirosawa, Igaya-cho, Kariya, Aichi 448-8542, Japan \\ ${ }^{8}$ National Institute of Technology, Wakayama College, 77 Noshima, Nada-cho, Gobo, Wakayama 644-0023, Japan
}

(Received 21 December 2019; accepted 23 January 2020; published 26 February 2020)

\begin{abstract}
Searching for space-time variations of the constants of Nature is a promising way to search for new physics beyond general relativity and the standard model motivated by unification theories and models of dark matter and dark energy. We propose a new way to search for a variation of the fine-structure constant using measurements of late-type evolved giant stars from the $\mathrm{S}$ star cluster orbiting the supermassive black hole in our Galactic Center. A measurement of the difference between distinct absorption lines (with different sensitivity to the fine structure constant) from a star leads to a direct estimate of a variation of the fine structure constant between the star's location and Earth. Using spectroscopic measurements of five stars, we obtain a constraint on the relative variation of the fine structure constant below $10^{-5}$. This is the first time a varying constant of nature is searched for around a black hole and in a high gravitational potential. This analysis shows new ways the monitoring of stars in the Galactic Center can be used to probe fundamental physics.
\end{abstract}

DOI: 10.1103/PhysRevLett.124.081101

The current understanding of our Universe is based on the theory of general relativity (GR) and on the standard model (SM) of particle physics. While both theories have been extremely successful, they are expected to break down at a certain point. In particular, a breaking of the Einstein equivalence principle is expected in various unification scenarios [1,2], in higher dimensional theories [3], and by some models of dark matter [4,5] and dark energy [6,7]. On a more philosophical note, the "principle of absence of absolute structure" led to many developments of extensions of physics where the constants of physics become dynamical entities, explicitly breaking the equivalence principle (see the discussion in section 2 of [8]).

One way to test the equivalence principle is to search for space-time variations of the constants of nature such as the fine structure constant $\alpha$, the mass of fermions and the quantum chromodynamics energy scale (see [9] for a review of the tests of GR and [10] for a review of the search for varying constants). Various experiments using atomic clocks have provided stringent constraints on linear drifts of the constants of nature at the level of $10^{-16} \mathrm{yr}^{-1}$ [11-18], on a dependency of the constants of nature to the Sun gravitational potential at the level of $10^{-7}[14-16,19,20]$ or on harmonic variations of the constants of nature [21]. A time variation of $\alpha$ has also been searched for using measurements of quasar absorption spectra [22] providing constraint on $\Delta \alpha / \alpha$ at the level of $10^{-6}$ for a cosmological redshift up to $z \sim 3$. A variation of $\alpha$ has also been constrained at the level of $10^{-3}$ for $z \sim 10^{3}$ using cosmic microwave background measurements [23] and at a similar level for $z \sim 10^{10}$ by a big bang nucleosynthesis analysis [24]. Finally, a dependency of $\alpha$ on the gravitational potential has also been searched for using absorption lines from a white dwarf [25]. Although many searches for a variation of $\alpha$ have been performed, the question of its constancy around a black hole and around a supermassive body remains totally open.

The motion of the short-period stars ( $\mathrm{S}$ stars) orbiting around the $4 \times 10^{6} M_{\odot}$ supermassive black hole (SMBH) at the center of our Galaxy has been monitored for 25 years by two experiments: one carried out at the Keck Observatory [26-28] and the other with the New Technology Telescope (NTT) and with the Very Large Telescope (VLT) [29-31]. Recently, these measurements have opened a new window to probe fundamental physics around a SMBH. Measurements of the short-period star 
S0-2/S2 have been used to search for a fifth interaction [27,32], to measure the relativistic redshift during its 2018 closest approach $[28,30]$ and to perform a null-redshift test [31].

In this Letter, we present a novel search for variations of the fine structure constant around a SMBH using spectroscopic measurements from late-type evolved giant stars orbiting Sagittarius A* $\left(\mathrm{Sgr} \mathrm{A}^{*}\right)$. This is the first search for a varying $\alpha$ around a compact object (the compactness of a celestial body can be characterized by $\Xi=G M / c^{2} R$ with $R$ as the body's radius) and very massive object, exploring a new region in the parameter space (Fig. 1). Probing the gravitational interaction in such a region is important to test for theories which exhibit screening mechanism in the solar system (see, e.g., [6,32-34]) or for theories that exhibit a scalarization mechanism around a black hole [35].

Using spectroscopy, we can very precisely measure the wavelengths of atomic lines, which can be used to measure potential variations in the fine structure constant. The spectroscopic observable is given by

$$
\frac{\Delta \lambda_{j}}{\lambda_{j}}=\frac{\lambda_{j}(z, \alpha)-\lambda_{j}\left(z=0, \alpha_{0}\right)}{\lambda_{j}\left(z=0, \alpha_{0}\right)}=z-k_{\alpha, j}(1+z) \frac{\Delta \alpha}{\alpha},
$$

where $\lambda_{j}(z, \alpha)$ is the measured central wavelength of an absorption line $j$ at the $\mathrm{GC}$ for a value of $\alpha$ potentially different from the one on Earth $\alpha_{0}$ while $\lambda_{j}\left(0, \alpha_{0}\right)$ is the wavelength for the same line measured in the lab. In this expression, $z$ is the traditional Doppler shift (which includes the Newtonian velocity along the line of sight and the relativistic corrections on the redshift) and the last term encodes the impact due to a variation of $\alpha$ on the measurements. This term depends directly on $k_{\alpha, j}$, the sensitivity of the transition $j$ to $\alpha$ and defined by

$$
d \ln \nu_{j}=k_{\alpha, j} d \ln \alpha,
$$

where $\nu_{j}=c / \lambda_{j}$ is the frequency of the absorption line $j$.
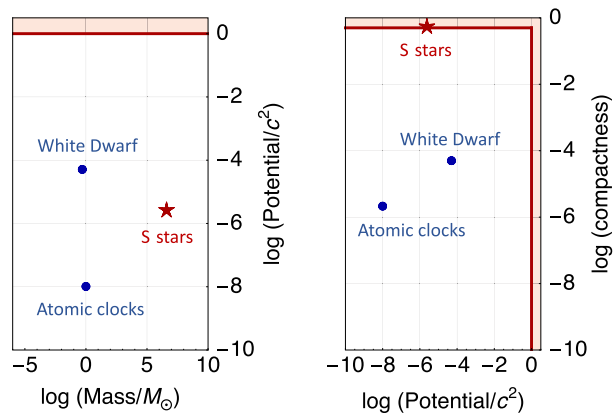

FIG. 1. Left: the gravitational potential probed by several searches for varying $\alpha$ against the mass of the central body that generates gravity in these tests. Right: the compactness $\Xi=$ $G M / c^{2} R$ of the central body probed by several searches for varying $\alpha$ against the gravitational potential. Searches for varying $\alpha$ with $\mathrm{S}$ stars explore a new region in this parameter space.
Late-type evolved stars are particularly useful for such an analysis because their cool atmospheres result in spectra that contain many strong atomic absorption lines which helps to maximize the number of different lines available for this analysis. Also importantly, their spectra contain lines that are related to atomic transitions with different sensitivity to $\alpha$ (the difference between the $k_{\alpha}$ coefficients are of the order of 1). In comparison, the more well studied young early-type $\mathrm{S}$ stars such as S0-2 have atmospheres that are very hot, so their near-infrared (NIR) spectra contain only a few $\mathrm{H}$ and He absorption. Unfortunately, $\mathrm{H}$ and $\mathrm{He}$ lines have nearly the same sensitivity to $\alpha$ (the difference between the $k_{\alpha}$ coefficients are of the order of $10^{-5}$ ) making these lines insensitive to the effect of variations in $\alpha$.

In this Letter, we focus on 5 late-type giants in orbit around the supermassive black hole: S0-6, S0-12, S0-13, S1-5, and S1-23, see Fig. 2 and [36]. These are among the closest late-type stars to the black hole $(0.4$ to 1.5 arcseconds in projection) with NIR spectroscopic measurements. Observations of these stars include imaging data from Keck Observatory and spectroscopic data from Gemini North, Keck Observatory, and Subaru telescope. The imaging data include both speckle imaging from the Near-Infrared Camera (NIRC) instrument (1995-2005) and adaptive optics data from the Near-Infrared Camera 2 (NIRC2) instrument (2005-2018). Details of the data reduction are given in [28]. NIR spectra were obtained with the Near-Infrared Integral Field Spectrometer (NIFS) instrument on Gemini North for the stars S0-6, S0-12, S0-13, and S1-5 using the K-band filter (0.95 to $2.40 \mu \mathrm{m}$ ) at a spectral resolution $R=\lambda / \Delta \lambda=5000$. We use spectra of these stars taken on May 13 and May 22, 2018. Additional spectroscopy of the star S0-6 was obtained

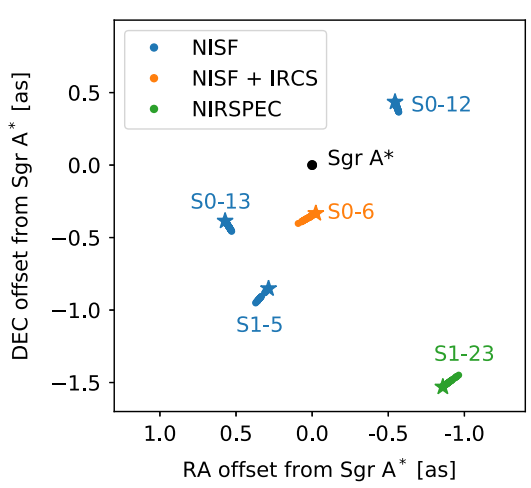

FIG. 2. Representation of the motion of the five stars considered in our analysis between 1995 and 2018. The large stars represent the positions of the stars in 2018. All these stars have a magnitude brighter than $M=15$. S0-6, S0-12, S0-13, S1-5 have been measured spectroscopically with the NIFS instrument with Gemini in 2018, S0-6 was also measured with the IRCS instrument from Subaru in 2018 and S1-23 with the NIRSPEC instrument at Keck in 2016. 

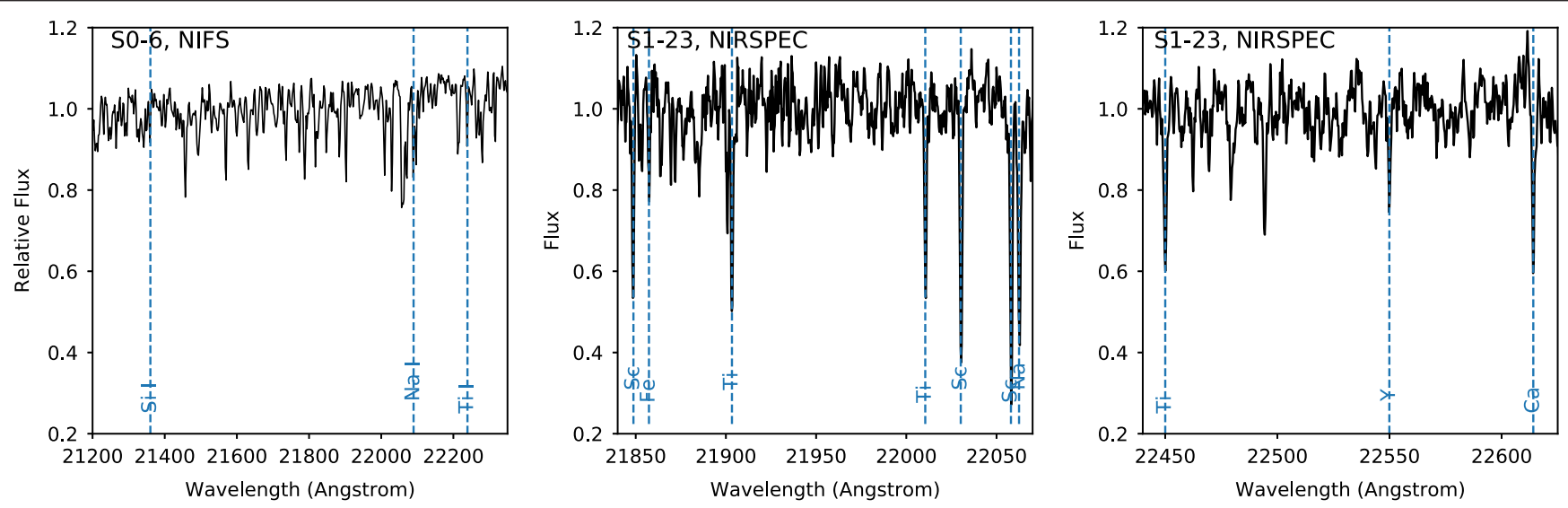

FIG. 3. Example spectra of the stars used in this work. Left: Spectrum of the star S0-6 observed using the NIFS spectrograph with a spectral resolution $R=5000$. We have identified three lines that are suitable for the fine structure constant analysis. Center and right: Spectra of the star S1-23 observed with the NIRSPEC instrument with $R=20000$. This higher spectral resolution allows us to identify ten atomic lines for use in this experiment.

using the Infrared Camera and Spectrograph (IRCS) instrument from Subaru. We use the order 26 spectrum (2.16 to $2.22 \mu \mathrm{m}$ ) at a resolution of $R=20000$ (down-sampled to $R=15000)$ for this work. Details of the data reduction for IRCS is given in [37] and [38]. Spectroscopy of the star S1-23 was obtained using the NIRSPEC instrument with laser-guide-star adaptive optics at Keck Observatory at a spectral resolution $R=20000$. NIRSPEC was used in echelle mode with the $\mathrm{K}$ filter. We use Orders 34 (2.24 to $2.27 \mu \mathrm{m}$ ) and 35 (2.18 to $2.21 \mu \mathrm{m}$ ) for this work. Details of the data reduction for the NIFS data and NIRSPEC data are given in [28] and [39], respectively. All the NIFS, IRCS, and NIRSPEC spectra have signal-to-noise ratios (SNR) greater than 20 in order to measure individual lines.

We identified a number of strong and sufficiently isolated absorption lines for this experiment (Fig. 3). In total, we measure three atomic lines from stars with NIFS spectra, six atomic lines with IRCS spectra, and ten atomic lines from the NIRSPEC spectra (Table I). The higher spectral resolution from NIRSPEC compared to NIFS allows us to use more lines. We use a Gaussian fit to determine the central wavelength of each atomic transition and a Monte Carlo simulation to estimate the uncertainty. The fitted lines and uncertainties are reported in Sec. II from the Supplemental Material [40].

To determine the sensitivity coefficients $k_{\alpha}$ for each absorption line used in this study, we perform $a b$ initio calculation of the spectrum for each element by solving the Dirac Hamiltonian in flat spacetime (curvature corrections are subdominant) and then compute $k_{\alpha}$ by a finite difference. The spectra are computed using a combination of the configuration interaction (CI) method with many-body perturbation theory (MBPT) [41-44] using the AMBiT software [45]. The full details of the calculations are presented in Sec. I from the Supplemental Material [40]; here we just outline briefly the main aspects of the method. The effective wave functions for a $M$-valence atom are expanded as a series of $M$-body Slater determinants, where the expansion coefficients are found variationally by minimizing the energy (CI method). To greatly improve the accuracy, the effects of core-valence electron correlations are also included into the valence wave functions. This is done by modifying the effective Hamiltonian for the valence states using MBPT before the CI procedure is performed. Finally, to calculate the $k_{\alpha}$ coefficients, we explicitly make small variations into value of the fine structure constant $(\alpha \rightarrow \alpha \pm \delta \alpha)$ in the code before the equations are solved.

Bayesian inference is used to estimate $\Delta \alpha / \alpha$ from the measurements and Eq. (1). The measurement errors for each line are assumed to be independent and to be normally distributed such that the full likelihood is the product of the individual likelihoods

$$
\mathcal{L}_{j} \propto \exp \left[-\frac{1}{2 \sigma_{j}^{2}}\left(\frac{\Delta \lambda_{j}}{\lambda_{j}}-z+k_{\alpha, j}(1+z) \frac{\Delta \alpha}{\alpha}\right)^{2}\right]
$$

where the subscript $j$ refers to a particular line. Uniform priors for $\Delta \alpha / \alpha$ and for $z$ are used and Gaussian priors are used for the sensitivity coefficients $k_{\alpha, j}$ with a mean value and an uncertainty quoted in Table I. We use the MultiNest sampler [70] to sample the posterior probability distribution function. All the epochs are fitted simultaneously but offsets between the different instruments or filters are taken into account (this results in different fitted velocities for each instrument or filter).

No significant deviation of the fine structure constant is detected for any of the stars considered in this analysis. The posterior probability distributions for each star can be found in Sec. IV from the Supplemental Material [40] and the $68 \%$ confidence intervals are reported in Table II. The constraints derived from the NIRSPEC measurements are one order of magnitude better than from the NIFS 
TABLE I. Atomic properties of the absorption lines used in this analysis. The wavelengths $\lambda$ are experimental values reported in [46]. The sensitivity to the fine structure constant $k_{\alpha}$ is computed from $a b$ initio calculation using the AMBiT software [45], see the discussion in Sec. I from the Supplemental Material [40]. The last column indicates which instrument has been used to measured each line with the following: (a) NIFS spectrograph, (b) IRCS spectrograph, (c) NIRSPEC order34, (d) NIRSPEC order35.

\begin{tabular}{|c|c|c|c|c|c|c|c|}
\hline \multirow[b]{2}{*}{${ }_{14} \mathrm{Si}$} & \multicolumn{2}{|c|}{ Lower } & \multicolumn{2}{|c|}{ Upper } & \multirow{2}{*}{$\frac{\lambda[\AA]}{21360.027}$} & \multirow{2}{*}{$\frac{k_{\alpha}}{0.013(9)}$} & \multirow{2}{*}{$\begin{array}{c}\text { instrument } \\
\mathrm{a}\end{array}$} \\
\hline & $3 s^{2} 3 p 4 p$ & ${ }^{1} D_{2}$ & $3 s^{2} 3 p 5 s$ & ${ }^{1} P_{1}^{o}$ & & & \\
\hline${ }_{11} \mathrm{NaNa}$ & $4 s$ & ${ }^{2} S_{1 / 2}$ & $4 p$ & ${ }^{2} P_{1 / 2}^{o}$ & 22089.728 & $0.004(2)$ & $a, b$ \\
\hline${ }_{22} \mathrm{Ti}$ & $3 d^{3} 4 s$ & ${ }^{5} P_{2}$ & $3 d^{2} 4 s 4 p$ & ${ }^{5} D_{2}^{o}$ & 22238.911 & $-0.34(10)$ & $\mathrm{a}$ \\
\hline${ }_{22} \mathrm{Ti}$ & $3 d^{3} 4 s$ & ${ }^{5} P_{2}$ & $3 d^{2} 4 s 4 p$ & ${ }^{5} D_{1}^{o}$ & 22450.025 & $-0.37(10)$ & $\mathrm{c}$ \\
\hline${ }_{9} \mathrm{Y}$ & $4 d^{2} 5 s$ & ${ }^{4} F_{7 / 2}$ & $4 d 5 s 5 p$ & ${ }^{4} F_{7 / 2}^{o}$ & 22549.938 & $-0.88(6)$ & $\mathrm{c}$ \\
\hline${ }_{20} \mathrm{Ca}$ & $4 s 4 d$ & ${ }^{3} D_{1}$ & $4 s 4 f$ & ${ }^{3} F_{2}^{o}$ & 22614.115 & $-0.03(1)$ & $\mathrm{c}$ \\
\hline${ }_{21}^{2} \mathrm{Sc}$ & $3 d^{2} 4 s$ & ${ }^{4} F_{3 / 2}$ & $3 d 4 s 4 p$ & ${ }^{2} D_{3 / 2}^{o}$ & 21848.743 & $-0.23(3)$ & $b, d$ \\
\hline${ }_{39} \mathrm{Fe}$ & $3 d^{6} 4 s^{2}$ & ${ }^{3} D_{3}$ & $3 d^{6} 4 s 4 p$ & ${ }^{3} P_{2}^{o}$ & 21857.345 & $0.56(28)$ & $\mathrm{d}$ \\
\hline${ }_{22} \mathrm{Ti}$ & $3 d^{3} 4 s$ & ${ }^{5} P_{2}$ & $3 d^{2} 4 s 4 p$ & ${ }^{5} D_{3}^{o}$ & 21903.353 & $-0.30(10)$ & $b, d$ \\
\hline${ }_{22}^{2} \mathrm{Ti}$ & $3 d^{3} 4 s$ & ${ }^{5} P_{1}$ & $3 d^{2} 4 s 4 p$ & ${ }^{5} D_{2}^{o}$ & 22010.501 & $-0.31(9)$ & $b, d$ \\
\hline${ }_{21}^{2} \mathrm{Sc}$ & $3 d^{2} 4 s$ & ${ }^{4} F_{5 / 2}$ & $3 d 4 s 4 p$ & ${ }^{2} D_{3 / 2}^{o}$ & 22030.179 & $-0.25(4)$ & $b, d$ \\
\hline${ }_{21} \mathrm{Sc}$ & $3 d^{2} 4 s$ & ${ }^{4} F_{9 / 2}$ & $3 d 4 s 4 p$ & ${ }^{4} D_{7 / 2}^{o}$ & 22058.003 & $-0.29(4)$ & $\mathrm{d}$ \\
\hline${ }_{11}^{2} \mathrm{Na}$ & $4 s$ & ${ }^{2} S_{1 / 2}$ & $4 p$ & ${ }^{2} P_{3 / 2}^{o}$ & 22062.485 & $0.007(2)$ & $b, d$ \\
\hline
\end{tabular}

instrument due to the better spectral resolution and more atomic lines observed with NIRSPEC.

A fit combining all the stars using a global likelihood (see Sec. IV from the Supplemental Material [40] for more details) provides a constraint of

$$
\frac{\Delta \alpha}{\alpha}=(1.0 \pm 5.8) \times 10^{-6},
$$

between the GC and Earth. This constraint is at the same level of magnitude as the ones obtained from quasar

TABLE II. $68 \%$ confidence interval for $\Delta \alpha / \alpha$ and for $z$ estimated from different stars. An estimation of the gravitational potential $U$ at the location of the star is also provided (see Sec. III from the Supplemental Material [40]). For S0-12, S0-13, and S1-5, two NIFS measurement epochs are combined with three absorption lines per epoch. S0-6 has been observed with NIFS and IRCS (each instrument has a different wavelength solution which reflects in an offset in their estimated $z$ value) providing nine lines. S1-23 has been observed with two different filters (each filter has a different wavelength solution which reflects in an offset in their estimated $z$ value) providing ten absorption lines.

\begin{tabular}{llll}
\hline \hline Star & \multicolumn{1}{c}{$\Delta \alpha / \alpha$} & \multicolumn{1}{c}{$U / c^{2}$} \\
\hline S0-6 & $(1.0 \pm 1.2) \times 10^{-4}$ & $71.0 \pm 10.4$ & $2.4 \times 10^{-6}$ \\
& & $-3.6 \pm 5.3$ & \\
S0-12 & $(-0.3 \pm 1.4) \times 10^{-4}$ & $-57.3 \pm 4.6$ & $1.6 \times 10^{-6}$ \\
S0-13 & $(0.03 \pm 3.5) \times 10^{-4}$ & $-61.6 \pm 18.1$ & $9.4 \times 10^{-7}$ \\
S1-5 & $(-0.7 \pm 2.4) \times 10^{-4}$ & $-3.7 \pm 19.4$ & $6.5 \times 10^{-7}$ \\
S1-23 & $(0.9 \pm 5.8) \times 10^{-6}$ & $-311.4 \pm 1.1$ & $4.6 \times 10^{-7}$ \\
& & $288.1 \pm 0.7$ & \\
\hline \hline
\end{tabular}

observations and is the first constraint on a possible variation of $\alpha$ around a $\mathrm{BH}$.

In several alternative theories of gravitation, the fine structure constant becomes dependent on the gravitational potential (see, e.g., [71]) and it is useful to consider the following parametrization

$$
\frac{\Delta \alpha}{\alpha}=\beta_{\alpha} \frac{\Delta U}{c^{2}},
$$

where $U$ is the Newtonian potential, $c$ the speed of light in a vacuum, and where $\beta_{\alpha}$ depends on the fundamental parameters of the theory. An estimate of the gravitational potential probed by the five stars considered in this analysis is required in order to constrain the $\beta_{\alpha}$ parameter. We infer the radial acceleration experienced by the stars using 25 years of astrometric measurements of the GC. Between 1995 and 2005, speckle imaging data provides astrometric diffraction-limited measurements of the central $5^{\prime \prime} \times 5^{\prime \prime}$ of the GC $[26,28,47]$. Between 2005 and 2018, adaptive optics (AO) imaging provides high-resolution images of the central $10^{\prime \prime} \times 10^{\prime \prime}$ of the GC $[26,28]$. AO allows for more efficient observations at the diffraction limit, resulting in measurements typically one order of magnitude better than speckle observations. These astrometric measurements are aligned [48] in a common reference frame defined by tying infrared observations of seven $\mathrm{SiO}$ masers $[49,72]$ to their radio counterpart [50]. The resulting 2D position measurements of the five stars considered in our analysis are provided in the Sec. III from the Supplemental Material [40]. A polynomial fit of these measurements give an estimate of the $3 \mathrm{D}$ radial 
acceleration of these stars which is transformed into an estimate of the gravitational potential using the SMBH mass $M=3.975 \times 10^{6} M_{\odot}$ reported in [28]. The estimate of the gravitational potential experienced by each star is reported in Table II. A fit combining the measurements from the five stars and using the estimate from the gravitational potential from Table II leads to

$$
\beta_{\alpha}=3.6 \pm 12.0
$$

at $68 \%$ confidence level. No deviation from GR is reported. This result is 8 orders of magnitude less constraining than a similar constraint obtained with atomic clocks around the Sun [20] and one order of magnitude less constraining than a similar result obtained around a white dwarf [25]. Nevertheless, this is the first time such a measurement is performed around a $\mathrm{BH}$ and around such a massive object (see Fig. 1). Such a measurement is particularly appropriate to constrain the presence of a scalar field around a $\mathrm{BH}$, which can naturally be enhanced in theories exhibiting $\mathrm{BH}$ scalarization mechanism, see, e.g., [35,73].

In conclusion, we propose a new method to search for a variation of the fine structure constant around a SMBH. Using existing measurements from NIFS spectrograph at GEMINI, from IRCS spectrograph from Subaru and from the NIRSPEC spectrograph at the Keck Observatory, we report a constraint on $\Delta \alpha / \alpha$ below $10^{-5}$ and a dependency of $\Delta \alpha / \alpha$ on the gravitational potential at the level of 10 . This is the first time such a search has been performed around a compact object, around a $\mathrm{BH}$ or around such a massive object $\left(\sim 4 \times 10^{6} M_{\odot}\right)$, see Fig. 1. The results reported in this Letter could be improved by dedicated measurement sessions using high resolution spectrograph to measure spectrum of late-type stars closer to the GC. For example, a dedicated measurement of S0-6's spectrum using NIRSPEC could improve the limit on $\beta_{\alpha}$ by one order of magnitude. Furthermore, it is expected that future spectrographs with advanced AO systems such as KAPA at Keck [74] would be able to measure spectrum of fainter late-type stars that are orbiting close to the GC (like, e.g., S0-38/S38 [26]), allowing us to improve significantly the current constraints (by up to 4 orders of magnitude) and allowing one to probe the fine structure constant in a higher gravitational potential. Finally, after the search for a fifth force [27,32], the relativistic redshift measurement of the star S0-2/S2 $[28,30]$ and a null test of the local position invariance using S0-2/S2 measurements [31], this analysis gives a new example of implications of GC measurements in term of fundamental physics.

We thank the staff and astronomers at Keck Observatory and Gemini Observatory, especially Randy Campbell and Terry Stickel for all their help in obtaining the new NIRSPEC data. Some of the data presented herein were obtained at the W. M. Keck Observatory, which is operated as a scientific partnership among the California Institute of
Technology, the University of California and the National Aeronautics and Space Administration and which was made possible by the generous financial support of the W. M. Keck Foundation. This work is partly based on observations obtained (Program ID GN-2018A-Q-123, PI: Do) at the Gemini Observatory, which is operated by the Association of Universities for Research in Astronomy, Inc., under a cooperative agreement with the NSF on behalf of the Gemini partnership: the National Science Foundation (United States), National Research Council (Canada), CONICYT (Chile), Ministerio de Ciencia, Tecnología Innovación Productiva (Argentina), Ministério da Ciência, Tecnologia e Inovacao (Brazil), and Korea Astronomy and Space Science Institute (Republic of Korea). This work is also based in part on data collected at Subaru Telescope, which is operated by the National Astronomical Observatory of Japan. S. N. was supported by JSPS KAKENHI, Grants No. JP15K13463, No. JP18K18760, and No. JP19H00695. Support for this work was provided by NSF AAG AST-1909554, the W. M. Keck Foundation, the Heising-Simons Foundation, and the Gordon and Betty Moore Foundation. Y. T. was supported by JSPS KAKENHI, Grant-in-Aid for Young Scientists (B) 26800150. We thank Julian Berengut for making the AMBiT software publicly available, and for discussions regarding its use.

*aurelien.hees@obpsm.fr

[1] T. R. Taylor and G. Veneziano, Phys. Lett. B 213, 450 (1988); T. Damour and A. M. Polyakov, Nucl. Phys. B423, 532 (1994); Gen. Relativ. Gravit. 26, 1171 (1994).

[2] P. Fayet, Phys. Rev. D 97, 055039 (2018); 99, 055043 (2019).

[3] I. Antoniadis, S. Dimopoulos, and G. Dvali, Nucl. Phys. B516, 70 (1998); V. A. Rubakov, Phys. Usp. 44, 871 (2001); R. Maartens and K. Koyama, Living Rev. Relativ. 13, 5 (2010); I. Antoniadis, S. Baessler, M. Büchner, V. V. Fedorov, S. Hoedl, A. Lambrecht, V. V. Nesvizhevsky, G. Pignol, K. V. Protasov, S. Reynaud, and Y. Sobolev, C.R. Phys. 12, 755 (2011).

[4] A. Arvanitaki, J. Huang, and K. Van Tilburg, Phys. Rev. D 91, 015015 (2015); Y. V. Stadnik and V. V. Flambaum, Phys. Rev. Lett. 115, 201301 (2015); A. Hees, O. Minazzoli, E. Savalle, Y. V. Stadnik, and P. Wolf, Phys. Rev. D 98, 064051 (2018).

[5] S. M. Carroll, S. Mantry, M. J. Ramsey-Musolf, and C. W. Stubbs, Phys. Rev. Lett. 103, 011301 (2009).

[6] J. Khoury and A. Weltman, Phys. Rev. Lett. 93, 171104 (2004); Phys. Rev. D 69, 044026 (2004).

[7] C. J. A. P. Martins and A. M. M. Pinho, Phys. Rev. D 91, 103501 (2015); C. J. A. P. Martins, A. M. M. Pinho, R. F. C. Alves, M. Pino, C. I. S. A. Rocha, and M. von Wietersheim, J. Cosmol. Astropart. Phys. 08 (2015) 047.

[8] T. Damour, Classical Quantum Gravity 29, 184001 (2012).

[9] C. M. Will, Living Rev. Relativ. 17, 4 (2014).

[10] J.-P. Uzan, Living Rev. Relativ. 14, 2 (2011). 
[11] H. Marion, F. P. Dos Santos, M. Abgrall, S. Zhang, Y. Sortais, S. Bize, I. Maksimovic, D. Calonico, J. Grünert, C. Mandache, P. Lemonde, G. Santarelli, P. Laurent, A. Clairon, and C. Salomon, Phys. Rev. Lett. 90, 150801 (2003).

[12] S. Bize, S. A. Diddams, U. Tanaka, C. E. Tanner, W. H. Oskay, R. E. Drullinger, T. E. Parker, T. P. Heavner, S. R. Jefferts, L. Hollberg, W. M. Itano, and J. C. Bergquist, Phys. Rev. Lett. 90, 150802 (2003).

[13] T. Rosenband, D. B. Hume, P. O. Schmidt, C. W. Chou, A. Brusch, L. Lorini, W. H. Oskay, R. E. Drullinger, T. M. Fortier, J. E. Stalnaker, S. A. Diddams, W. C. Swann, N. R. Newbury, W. M. Itano, D. J. Wineland, and J. C. Bergquist, Science 319, 1808 (2008).

[14] J. Guéna, M. Abgrall, D. Rovera, P. Rosenbusch, M. E. Tobar, P. Laurent, A. Clairon, and S. Bize, Phys. Rev. Lett. 109, 080801 (2012).

[15] M. E. Tobar, P. L. Stanwix, J. J. McFerran, J. Guéna, M. Abgrall, S. Bize, A. Clairon, P. Laurent, P. Rosenbusch, D. Rovera, and G. Santarelli, Phys. Rev. D 87, 122004 (2013).

[16] N. Leefer, C. T. M. Weber, A. Cingöz, J. R. Torgerson, and D. Budker, Phys. Rev. Lett. 111, 060801 (2013).

[17] R. M. Godun, P. B. R. Nisbet-Jones, J. M. Jones, S. A. King, L. A. M. Johnson, H. S. Margolis, K. Szymaniec, S. N. Lea, K. Bongs, and P. Gill, Phys. Rev. Lett. 113, 210801 (2014).

[18] N. Huntemann, B. Lipphardt, C. Tamm, V. Gerginov, S. Weyers, and E. Peik, Phys. Rev. Lett. 113, 210802 (2014).

[19] S. Peil, S. Crane, J. L. Hanssen, T. B. Swanson, and C. R. Ekstrom, Phys. Rev. A 87, 010102(R) (2013).

[20] N. Ashby, T. E. Parker, and B. R. Patla, Nat. Phys. 14, 822 (2018).

[21] K. Van Tilburg, N. Leefer, L. Bougas, and D. Budker, Phys. Rev. Lett. 115, 011802 (2015); A. Hees, J. Guéna, M. Abgrall, S. Bize, and P. Wolf, Phys. Rev. Lett. 117, 061301 (2016).

[22] J. K. Webb, V. V. Flambaum, C. W. Churchill, M. J. Drinkwater, and J. D. Barrow, Phys. Rev. Lett. 82, 884 (1999); J. K. Webb, M. T. Murphy, V. V. Flambaum, V. A. Dzuba, J. D. Barrow, C. W. Churchill, J. X. Prochaska, and A. M. Wolfe, Phys. Rev. Lett. 87, 091301 (2001); M. T. Murphy, J. K. Webb, V. V. Flambaum, V. A. Dzuba, C. W. Churchill, J. X. Prochaska, J. D. Barrow, and A. M. Wolfe, Mon. Not. R. Astron. Soc. 327, 1208 (2001); J. K. Webb, M. T. Murphy, V. V. Flambaum, and S. J. Curran, Astrophys. Space Sci. 283, 565 (2003); M. T. Murphy, J. K. Webb, and V. V. Flambaum, Mon. Not. R. Astron. Soc. 345, 609 (2003); R. Srianand, H. Chand, P. Petitjean, and B. Aracil, Phys. Rev. Lett. 92, 121302 (2004); H. Chand, R. Srianand, P. Petitjean, and B. Aracil, Astron. Astrophys. 417, 853 (2004); P. Tzanavaris, J. K. Webb, M. T. Murphy, V. V. Flambaum, and S. J. Curran, Phys. Rev. Lett. 95, 041301 (2005); P. Tzanavaris, M. T. Murphy, J. K. Webb, V. V. Flambaum, and S. J. Curran, Mon. Not. R. Astron. Soc. 374, 634 (2007); M. T. Murphy, J. K. Webb, and V. V. Flambaum, Mon. Not. R. Astron. Soc. 384, 1053 (2008); R. Srianand, P. Noterdaeme, C. Ledoux, and P. Petitjean, Astron. Astrophys. 482, L39 (2008); P. Petitjean, R. Srianand, H. Chand, A. Ivanchik, P. Noterdaeme, and N. Gupta, Space Sci. Rev. 148, 289 (2009); J. K. Webb, J. A. King, M. T. Murphy, V. V. Flambaum, R. F. Carswell, and
M. B. Bainbridge, Phys. Rev. Lett. 107, 191101 (2011); J. A. King, J. K. Webb, M. T. Murphy, V. V. Flambaum, R. F. Carswell, M. B. Bainbridge, M. R. Wilczynska, and F. E. Koch, Mon. Not. R. Astron. Soc. 422, 3370 (2012).

[23] P. A. R. Ade et al. (Planck Collaboration), Astron. Astrophys. 571, A16 (2014).

[24] P. P. Avelino, S. Esposito, G. Mangano, C. J. A. P. Martins, A. Melchiorri, G. Miele, O. Pisanti, G. Rocha, and P. T. P. Viana, Phys. Rev. D 64, 103505 (2001).

[25] J. C. Berengut, V. V. Flambaum, A. Ong, J. K. Webb, J. D. Barrow, M. A. Barstow, S. P. Preval, and J. B. Holberg, Phys. Rev. Lett. 111, 010801 (2013).

[26] A. M. Ghez, B. L. Klein, M. Morris, and E. E. Becklin, Astrophys. J. 509, 678 (1998); A. M. Ghez, M. Morris, E. E. Becklin, A. Tanner, and T. Kremenek, Nature (London) 407, 349 (2000); A. M. Ghez, G. Duchêne, K. Matthews, S. D. Hornstein, A. Tanner, J. Larkin, M. Morris, E. E. Becklin, S. Salim, T. Kremenek, D. Thompson, B. T. Soifer, G. Neugebauer, and I. McLean, Astrophys. J. Lett. 586, L127 (2003); A. M. Ghez, S. Salim, S. D. Hornstein, A. Tanner, J. R. Lu, M. Morris, E. E. Becklin, and G. Duchêne, Astrophys. J. 620, 744 (2005); A. M. Ghez, S. D. Hornstein, J. R. Lu, A. Bouchez, D. Le Mignant, M. A. van Dam, P. Wizinowich, K. Matthews, M. Morris, E. E. Becklin, R. D. Campbell, J. C. Y. Chin, S. K. Hartman, E. M. Johansson, R. E. Lafon, P. J. Stomski, and D. M. Summers, Astrophys. J. 635, 1087 (2005); A. M. Ghez, S. Salim, N. N. Weinberg, J. R. Lu, T. Do, J. K. Dunn, K. Matthews, M. R. Morris, S. Yelda, E. E. Becklin, T. Kremenek, M. Milosavljevic, and J. Naiman, Astrophys. J. 689, 1044 (2008); L. Meyer, A. M. Ghez, R. Schödel, S. Yelda, A. Boehle, J. R. Lu, T. Do, M. R. Morris, E. E. Becklin, and K. Matthews, Science 338, 84 (2012); A. Boehle, A. M. Ghez, R. Schödel, L. Meyer, S. Yelda, S. Albers, G. D. Martinez, E. E. Becklin, T. Do, J. R. Lu, K. Matthews, M. R. Morris, B. Sitarski, and G. Witzel, Astrophys. J. 830, 17 (2016); D. S. Chu, T. Do, A. Hees, A. Ghez, S. Naoz, G. Witzel, S. Sakai, S. Chappell, A. K. Gautam, J. R. Lu, and K. Matthews, Astrophys. J. 854, 12 (2018).

[27] A. Hees, T. Do, A. M. Ghez, G. D. Martinez, S. Naoz, E. E. Becklin, A. Boehle, S. Chappell, D. Chu, A. Dehghanfar, K. Kosmo, J. R. Lu, K. Matthews, M. R. Morris, S. Sakai, R. Schödel, and G. Witzel, Phys. Rev. Lett. 118, 211101 (2017).

[28] T. Do, A. Hees, A. M. Ghez, G. Martinez, D. Chu et al., Science 365, 664 (2019).

[29] R. Genzel, A. Eckart, T. Ott, and F. Eisenhauer, Mon. Not. R. Astron. Soc. 291, 219 (1997); A. Eckart and R. Genzel, Mon. Not. R. Astron. Soc. 284, 576 (1997); R. Schödel, T. Ott, R. Genzel, R. Hofmann, M. Lehnert, A. Eckart, N. Mouawad, T. Alexander, M. J. Reid, R. Lenzen, M. Hartung, F. Lacombe, D. Rouan, E. Gendron, G. Rousset, A.-M. Lagrange, W. Brandner, N. Ageorges, C. Lidman, A. F. M. Moorwood, J. Spyromilio, N. Hubin, and K. M. Menten, Nature (London) 419, 694 (2002); A. Eckart, R. Genzel, T. Ott, and R. Schödel, Mon. Not. R. Astron. Soc. 331, 917 (2002); F. Eisenhauer, R. Schödel, R. Genzel, T. Ott, M. Tecza, R. Abuter, A. Eckart, and T. Alexander, Astrophys. J. Lett. 597, L121 (2003); F. Eisenhauer, R. Genzel, T. Alexander, R. Abuter, T. Paumard, T. Ott, 
A. Gilbert, S. Gillessen, M. Horrobin, S. Trippe, H. Bonnet, C. Dumas, N. Hubin, A. Kaufer, M. Kissler-Patig, G. Monnet, S. Ströbele, T. Szeifert, A. Eckart, R. Schödel, and S. Zucker, Astrophys. J. 628, 246 (2005); S. Gillessen, F. Eisenhauer, T. K. Fritz, H. Bartko, K. Dodds-Eden, O. Pfuhl, T. Ott, and R. Genzel, Astrophys. J. Lett. 707, L114 (2009); S. Gillessen, F. Eisenhauer, S. Trippe, T. Alexander, R. Genzel, F. Martins, and T. Ott, Astrophys. J. 692, 1075 (2009); S. Gillessen, P. M. Plewa, F. Eisenhauer, R. Sari, I. Waisberg, M. Habibi, O. Pfuhl, E. George, J. Dexter, S. von Fellenberg, T. Ott, and R. Genzel, Astrophys. J. 837, 30 (2017); R. Abuter et al. (Gravity Collaboration), Astron. Astrophys. 625, L10 (2019).

[30] R. Abuter et al. (Gravity Collaboration), Astron. Astrophys. 615, L15 (2018).

[31] A. Amorim et al. (Gravity Collaboration), Phys. Rev. Lett. 122, 101102 (2019).

[32] D. Borka, P. Jovanović, V. Borka Jovanović, and A. F. Zakharov, J. Cosmol. Astropart. Phys. 11 (2013) 050; A. F. Zakharov, P. Jovanović, D. Borka, and V. Borka Jovanović, J. Cosmol. Astropart. Phys. 05 (2016) 045; 04 (2018) 050 .

[33] K. Hinterbichler and J. Khoury, Phys. Rev. Lett. 104, 231301 (2010); K. Hinterbichler, J. Khoury, A. Levy, and A. Matas, Phys. Rev. D 84, 103521 (2011).

[34] A. I. Vainshtein, Phys. Lett. B 39, 393 (1972); C. Deffayet, G. Dvali, G. Gabadadze, and A. Vainshtein, Phys. Rev. D 65, 044026 (2002).

[35] D. D. Doneva and S. S. Yazadjiev, Phys. Rev. Lett. 120, 131103 (2018); G. Antoniou, A. Bakopoulos, and P. Kanti, Phys. Rev. Lett. 120, 131102 (2018); H. O. Silva, J. Sakstein, L. Gualtieri, T. P. Sotiriou, and E. Berti, Phys. Rev. Lett. 120, 131104 (2018).

[36] T. Do, J. R. Lu, A. M. Ghez, M. R. Morris, S. Yelda, G. D. Martinez, S. A. Wright, and K. Matthews, Astrophys. J. 764, 154 (2013).

[37] S. Nishiyama, H. Saida, Y. Takamori, M. Takahashi, R. Schödel, F. Najarro, S. Hamano, M. Omiya, M. Tamura, M. Takahashi, H. Gorin, S. Nagatomo, and T. Nagata, Publ. Astron. Soc. Jpn. 70, 74 (2018).

[38] H. Saida, S. Nishiyama, T. Ohgami, Y. Takamori, M. Takahashi, Y. Minowa, F. Najarro, S. Hamano, M. Omiya, A. Iwamatsu, M. Takahashi, H. Gorin, T. Kara, A. Koyama, Y. Ohashi, M. Tamura, S. Nagatomo, T. Zenko, and T. Nagata, Publ. Astron. Soc. Jpn. 71, 126 (2019).

[39] T. Do, W. Kerzendorf, Q. Konopacky, J. M. Marcinik, A. Ghez, J. R. Lu, and M. R. Morris, Astrophys. J. Lett. 855, L5 (2018).

[40] See the Supplemental Material at http://link.aps.org/ supplemental/10.1103/PhysRevLett.124.081101 for the detailed calculation of the atomic transitions, for the data and for a details presentation of the methodology used in this analysis, which includes Refs. [26,28,37,39,41-69].

[41] V. A. Dzuba, V. V. Flambaum, and M. G. Kozlov, Phys. Rev. A 54, 3948 (1996); V. A. Dzuba and W. R. Johnson, Phys. Rev. A 57, 2459 (1998).

[42] J. C. Berengut, Phys. Rev. A 94, 012502 (2016).

[43] V. A. Dzuba, J. C. Berengut, C. Harabati, and V. V. Flambaum, Phys. Rev. A 95, 012503 (2017).
[44] A. J. Geddes, L. V. Skripnikov, A. Borschevsky, J. C. Berengut, V. V. Flambaum, and T. P. Rakitzis, Phys. Rev. A 98, 022508 (2018).

[45] E. V. Kahl and J. C. Berengut, Comput. Phys. Commun. 238, 232 (2019).

[46] A. Kramida, Y. Ralchenko, J. Reader, and (The NIST ASD Team 2018), NIST Atomic Spectra Database (ver. 5.6.1), http://physics.nist.gov/asd (2019).

[47] Z. Chen, E. Gallego-Cano, T. Do, G. Witzel, A. M. Ghez, R. Schödel, B. N. Sitarski, E. E. Becklin, J. Lu, M. R. Morris, A. Dehghanfar, A. K. Gautam, A. Hees, M. W. Hosek, Jr., S. Jia, A. C. Mangian, and K. Matthews, Astrophys. J. Lett. 882, L28 (2019).

[48] S. Jia, J. R. Lu, S. Sakai, A. K. Gautam, T. Do, M. W. Hosek, Jr., M. Service, A. M. Ghez, E. Gallego-Cano, R. Schödel, A. Hees, M. R. Morris, E. Becklin, and K. Matthews, Astrophys. J. 873, 9 (2019).

[49] S. Sakai, J. R. Lu, A. Ghez, S. Jia, T. Do, G. Witzel, A. K. Gautam, A. Hees, E. Becklin, K. Matthews, and M. W. Hosek, Jr., Astrophys. J. 873, 65 (2019).

[50] M. J. Reid, K. M. Menten, S. Trippe, T. Ott, and R. Genzel, Astrophys. J. 659, 378 (2007).

[51] J. C. Berengut, V. A. Dzuba, V. V. Flambaum, and A. Ong, Phys. Rev. Lett. 109, 070802 (2012).

[52] M. S. Safronova, Ann. Phys. (N.Y.) 531, 1800364 (2019).

[53] M. G. Kozlov and D. Budker, Ann. Phys. (N.Y.) 531, 1800254 (2018).

[54] V. A. Dzuba, V. V. Flambaum, and M. G. Kozlov, Phys. Rev. A 99, 032501 (2019).

[55] B. G. C. Lackenby, V. A. Dzuba, and V. V. Flambaum Phys. Rev. A 99, 042509 (2019).

[56] J. C. Berengut, V. V. Flambaum, and M. G. Kozlov, Phys. Rev. A 73, 012504 (2006).

[57] J. C. Berengut, V. V. Flambaum, and M. G. Kozlov, J. Phys. B 41, 235702 (2008).

[58] V. A. Dzuba, Phys. Rev. A 71, 032512 (2005).

[59] V. V. Flambaum and V. A. Dzuba, Can. J. Phys. 87, 25 (2009).

[60] V. A. Dzuba and V. V. Flambaum, Phys. Rev. A 77, 012515 (2008).

[61] V. A. Dzuba, V. V. Flambaum, P. G. Silvestrov, and O. P. Sushkov, J. Phys. B 20, 1399 (1987).

[62] V. A. Dzuba, V. V. Flambaum, and O. P. Sushkov, Phys. Lett. A 140, 493 (1989).

[63] J. C. Berengut, V. A. Dzuba, V. V. Flambaum, and M. V. Marchenko, Phys. Rev. A 70, 064101 (2004).

[64] M. Støstad, T. Do, N. Murray, J. R. Lu, S. Yelda, and A. Ghez, Astrophys. J. 808, 106 (2015).

[65] R. Schödel, S. Yelda, A. Ghez, J. H. Girard, L. Labadie, R. Rebolo, A. Pérez-Garrido, and M. R. Morris, Mon. Not. R. Astron. Soc. 429, 1367 (2013).

[66] E. Diolaiti, O. Bendinelli, D. Bonaccini, L. Close, D. Currie, and G. Parmeggiani, Astron. Astrophys. 147, 335 (2000).

[67] P. L. Wizinowich, D. Le Mignant, A. H. Bouchez, R. D. Campbell, J. C. Y. Chin, A. R. Contos, M. A. van Dam, S. K. Hartman, E. M. Johansson, R. E. Lafon, H. Lewis, P. J. Stomski, D. M. Summers, C. G. Brown, P. M. Danforth, C. E. Max, and D. M. Pennington, Publ. Astron. Soc. Pac. 118, 297 (2006). 
[68] M. A. van Dam, A. H. Bouchez, D. Le Mignant, E. M. Johansson, P. L. Wizinowich, R. D. Campbell, J. C. Y. Chin, S. K. Hartman, R. E. Lafon, P. J. Stomski, Jr., and D. M. Summers, Publ. Astron. Soc. Pac. 118, 310 (2006).

[69] M. P. Hobson, G. P. Efstathiou, and A. N. Lasenby, General Relativity: An Introduction for Physicists (Cambridge University Press, Cambridge, 2006).

[70] F. Feroz and M. P. Hobson, Mon. Not. R. Astron. Soc. 384, 449 (2008); 398, 1601 (2009).

[71] J. D. Bekenstein, Phys. Rev. D 25, 1527 (1982); H. B. Sandvik, J. D. Barrow, and J. Magueijo, Phys. Rev. Lett. 88, 031302 (2002); A. Hees and O. Minazzoli, arXiv:1512 .05233 .
[72] S. Yelda, J. R. Lu, A. M. Ghez, W. Clarkson, J. Anderson, T. Do, and K. Matthews, Astrophys. J. 725, 331 (2010); S. Yelda, L. Meyer, A. Ghez, and T. Do, in Proceedings of the Third AO4ELT Conference, edited by S. Esposito and L. Fini (INAF, Firenze, 2013), p. 83.

[73] A. Hees, O. Minazzoli, E. Savalle, Y. V. Stadnik, P. Wolf, and B. M. Roberts, in Proceedings of the 52nd Rencontres de Moriond-Gravitation Session (ARISF, Paris, 2019).

[74] P. Wizinowich, J. Chin, K. Casey, S. Cetre, C. Correia, L. Hunter, S. Lilley, J. Lu, S. Ragland, E. Wetherell, A. Ghez, T. Do, T. Jones, M. Liu, D. Mawet, C. Max, M. Morris, T. Treu, and T. Wright, in Proceedings of the Sixth AO4ELT Conference (2019). 